\title{
O equilíbrio e a estrutura estelar em uma abordagem simples: a Seqüência Principal
}

\author{
Stellar equilibrium and structure from a simple approach: the Main Sequence
}

\author{
M. Bandecchi ${ }^{1}$, P.S. Bretones ${ }^{2}$, J.E. Horvath*1] \\ ${ }^{1}$ Departamento de Astronomia, Instituto de Astronomia, Geofísica e Ciências Atmosféricas da Universidade de São Paulo, São \\ Paulo,SP, Brasil \\ ${ }^{2}$ Departamento de Metodologia de Ensino, Universidade Federal de São Carlos, São Carlos, SP, Brasil
}

Recebido em 08 de Fevereiro, 2019. Revisado em 23 de Março, 2019. Aceito em 23 de Abril, 2019

\begin{abstract}
As condições simultâneas de equilíbrio Virial e equilíbrio hidrostático determinam a existência de soluções para os modelos estelares mais simples. Utilizando estes dois conceitos mostramos que é possível caracterizar a estrutura estelar sem recorrer à integração de equações diferenciais, ao menos para obter as propriedades globais. Além disso, discutimos como é que as estrelas geram energia, enfatizando o papel fundamental do decaimento fraco do próton que controla suas vidas e as impede que explodam em frações de segundo, colocando a teoria das interações fracas em um contexto muito mais visível e integrado para o estudante e seus professores. Pela sua abrangência e simplicidade, esta abordagem é particularmente adequada para apresentar estes temas no Ensino Médio.
\end{abstract}

Palavras-chave: equilíbrio Virial, estrelas, equilíbrio hidrostático.

\begin{abstract}
The simultaneous occurrence of Virial and hydrostatic equilibrium determine the existence of solutions for the simplest stellar models. Using these concepts we show how it is possible to characterize the stellar structure without having to integrate the relevant differential equations, at least to obtain global properties. Later we discuss how the stars generate energy. emphasizing the fundamental role of the weak decay of protons controlling the star lifetime, precluding their explosions in a fraction of a second, thus putting weak interactions on an integrated and more apparent context for the students and teachers. Because its generality and simplicity, this approach is particularly useful for the discussion in the Middle / High School.
\end{abstract}

Keywords: Virial equilibrium, stars, hydrostatic equilibrium.

\section{Introdução}

Em um artigo recente [1] discutimos recursos didáticos para apresentar em sala de aula alguns tópicos de Astrofísica Estelar, tema que, embora muito rico, formativo e integrador, rara vez chega concretamente a ser exposto pelos professores. As razões para este fato são várias e constituiriam um artigo à parte, mas para algumas considerações gerais nos remetemos ao trabalho antes mencionado [1] e suas referências. Um fato notório é que os próprios documentos oficiais, os PCN [2], nos conteúdos do Ensino Fundamentalcolocam a "evolução das estrelas, anãs brancas, gigantes vermelhas, estrelas de nêutrons e buracos negros"como temas a serem tratados. Mais ainda, a Proposta Curricular do estado de São Paulo [3] inclui nos conteúdos da disciplina de Física para Ensino Médio, o tema "As etapas da evolução estelar (formação, gigante vermelha, anã branca, supernova)". É claro que estas inclusões evidenciam uma avaliação positiva da introdução destes temas estelares, embora não sejam explícitos os caminhos da formação, a evolução

*Endereço de correspondência: foton@iag.usp.br. e o fim das estrelas nem os aspectos físicos envolvidos, os quais ocupam considerável espaço em livros didáticos [4] ou paradidáticos [5] de ampla circulação. A pouca inserção da pesquisa em educação em Astronomia foi também apontada no nosso trabalho anterior [1], e nos referimos a ele para ulteriores informações e referências, tanto no âmbito do Brasil quanto no internacional. Os trabalhos de Polanco Erazo [6] na Colômbia e Bandecchi [7] no Brasil são algumas das poucas tentativas de "fusionar"a Astrofísica Estelar com a Física do Ensino Médio, apresentando-a como uma síntese e um paradigma a ser introduzido com vantagens aos alunos dessas faixas etárias. Em outras palavras, existem vantagens notórias na discussão das estrelas como exemplos da Física do Ensino Médio, e acreditamos que isto possa reforçar e contextualizar o ensino dos fenômenos básicos da Mecânica, Eletromagnetismo, Termodinâmica e outros que já estão inseridos nos curricula em vigência.

O objetivo principal do nosso trabalho precedente [1] foi o de mostrar como a manutenção simultânea do equilíbrio Virial (associado à partilha da energia) e equilíbrio hidrostático (balanço entre a gravitação e as forças de 
pressão) levam a concluir que existe uma massa máxima para as estrelas compostas de gás e radiação, e que aquela precisamente acontece quando esta última componente começa a dominar a pressão total. Os valores obtidos para $M_{\max }$ com esta estimativa, entre $100-200 M_{\odot}$ coincidem dentro de um fator numérico da ordem da unidade com as observações das maiores massas de estrelas reais 1 Vamos abordar agora a descrição das estrelas mais básica utilizando de novo estes conceitos de equilíbrio, e discutir alguns fatos importantes das reações nucleares que sustentam a estrutura estelar.

Acreditamos que estes conceitos possam ser transmitidos de forma efetiva aos alunos do Ensino Médio, em linha com as sugestões curriculares antes mencionadas. Veremos também o efeito integrador da discussão qualitativa e quantitativa da Astrofísica Estelar de forma prática e acessível para o professor em sala de aula.

\section{A relação massa-raio das estrelas e quantidades decorrentes das relações de equilíbrio}

Como é bem sabido, a equação fundamental que estabelece o estado mecânico do gás é a chamada equação de equilíbrio hidrostático para uma esfera auto-gravitante

$$
\frac{d P}{d r}=-\frac{G m \rho}{r^{2}}
$$

onde $m(r)$ é a massa encerrada no raio $r$. A solução da equação (1) em termos da função pressão $P(r)$ estabelece que a estrela está sujeita aobalançode forças de pressão e a gravitação, escrita genericamente como $\sum P_{i}=$ "P $P_{\text {grav }}$ "na Ref. [1], onde a "pressão gravitacional"foi identificada com o efeito de variar a energia arespeito do volume que esta ocupa [8],de forma totalmente geral.

Embora o procedimento rigoroso consista na integração da equação (1) conjuntamente com outras similares que determinam toda a estrutura das estrelas, nos textos de ensino superior (por exemplo, [9]) a discussão das soluções da equação 11 começa geralmente com a ordem de grandeza e dependência da pressão $P(r)$ com as variáveis físicas (raio, massa, densidade) convertendo a equação diferencial em uma equação de incrementos finitos. Este procedimento pode (e deve) ser trasladado à sala de aula do Ensino Médio já que dispensa os conceitos do cálculo, e mesmo assim evidencia quantitativamente fatos importantes da estrutura estelar. O procedimento padrão é o de substituir diferenciais por incrementos, no caso os máximos incrementos possíveis (ou seja, $\Delta R=R-\mathrm{o}$ incremento entre o raio no centro $(0)$ e a superfície da estrela $(R)$ etc.). Desta forma a equação (1) se converte

\footnotetext{
${ }^{1}$ Ao longo deste trabalho as massas são medidas/referenciadas à massa do Sol, $M_{\odot}=1,99 \times 10^{30} \mathrm{~kg}$, assim como as luminosidades à luminosidade solar $L_{\odot}=4 \times 10^{33} \mathrm{ergs}^{-1}=3,83 \times 10^{26} \mathrm{~W}$ e os raios ao raio solar, $R_{\odot}=6,96 \times 10^{8} \mathrm{~m}[9]$.
}

em

$$
P_{c}-0=\frac{G(M-0)\left(\rho_{c}-0\right) \Delta R}{\Delta R^{2}} \rightarrow P_{c}=\frac{G M \rho_{c}}{R}
$$

já que a pressão na superfície estelar é nula por definição, enquanto toda a massa $M$ está incluída quando chegarmos à superfície. Com a hipótese adicional $\rho_{c}=$ cte $=3 M / 4 \pi R^{3}$, chegamos a estimativa usual que mostra a pressão central $P_{c} \propto \frac{M^{2}}{R^{4}}$. O mesmo procedimento aproximado na equação da conservação da massa

$$
\frac{d M}{d r}=4 \pi r^{2} \rho
$$

leva de imediato a

$$
M-0=4 \pi \Delta R^{2} \Delta R\left(\rho_{c}-0\right) \rightarrow M=4 \pi R^{3} \rho_{c}
$$

onde a falta do fator "3"usual no denominador é devida, obviamente, ao fato de não termos feito uma integração genuína da equação (3). Em todas estas estimativas fatores numéricos tais como $2, \pi$ etc. são costumeiramente ignorados em favor de uma transparência nos procedimentos que resultam em uma melhor visualização da relação entre a física do problema e sua implementação matemática, coisa que consideramos da maior importância para alunos do Ensino Médio e que facilita muito a tarefa do professor.

A equação (4) parece bastante simples, mas já permite uma compreensão importante da estrutura estelar. É evidente que se considerarmos $\rho_{c}=$ cte, então o raio estelar deve crescer como $R \propto M^{1 / 3}$. Mas esta última é uma hipótese muito forte, é de esperar que a densidade caia substancialmente para fora, até atingir um zero na superfície. Uma queda deste tipo, por exemplo $\rho \propto 1 / r$ leva de imediato, substituindo na equação (4) a $R \propto M^{1 / 2}$. Esta diferença pode ser convertida em um teste simples, já que existem várias estrelas da Seqüência Principal com raios e massas bem medidos.

Os dados de massas e raios para o conjunto de estrelas listado na Tabela 1 são mostrados na curva preta (Fig.1), enquanto a relação massa-raio que supõe uma queda na densidade $\rho \propto 1 / r$ ajusta muito bem (acima de $\sim 18 M_{\odot}$ existe uma "quebra"neste plano, produto do fato que por volta desse valor as estrelas são totalmente convectivas, mas o transporte de energia não foi aqui considerado em favor da simplicidade e preferimos nos limitar a estrelas abaixo desse valor limiar). A hipótese de $\rho_{c}=$ cte levaria a uma dependência bem mais forte $M \propto R^{3}$, em bastante desacordo com os dados. Assim, concluímos que deve existir uma queda importante na densidade estelar desde o centro até a superfície.

Podemos agora combinar esta informação com a relação de equilíbrio Virial, que indica como a matéria estabelece a partilha entre a energia interna $E_{\text {int }} \mathrm{e}$ a gravitacional $E_{\text {grav }}$

$$
E_{\text {grav }}+2 E_{\text {int }}=0
$$


Tabela 1: O conjunto de estrelas com massas e raios medidos e $\bar{T}$ calculadas com a equação (6). Existem algumas incertezas nas medidas individuais que não afetam substancialmente a conclusão $\rho \neq \rho_{c}$, apontando para uma função decrescente com o raio.

\begin{tabular}{|c|c|c|c|c|c|c|}
\hline Nome & $\begin{array}{c}\text { Tipo } \\
\text { Espectral }\end{array}$ & $\operatorname{Massa}\left(M_{\odot}\right)$ & Raio $\left(R_{\odot}\right)$ & $\begin{array}{c}\text { Temperatura } \\
\text { média } \bar{T}\left(10^{6} K\right)\end{array}$ & $\begin{array}{c}\text { Temperatura } \\
\text { efetiva } T_{\text {eff }}\left(10^{3} \mathrm{~K}\right)\end{array}$ & $\begin{array}{l}\text { Luminosidade } \\
\qquad\left(L_{\odot}\right)\end{array}$ \\
\hline$\varphi^{1}$ Orionis & B0 & 15,5 & 6,4 & 5,3 & 30 & 28800 \\
\hline$\beta$ Lyræ Aa1 & B1 & 13 & 6 & 4,8 & 30 & 26000 \\
\hline 1 Scorpii & $\mathrm{B} 2$ & 8,3 & 3,7 & 4,9 & 24 & 3900 \\
\hline$\pi$ Andromedae A & B5 & 6,5 & 3,8 & 3,4 & 12 & 1136 \\
\hline$\alpha$ Coronæ Borealis $\mathrm{A}$ & $\mathrm{A} 0$ & 3,2 & 2,5 & 2,8 & 9,7 & 74 \\
\hline$\beta$ Pictoris & A5 & 2,1 & 1,7 & 2,7 & 8 & 8,7 \\
\hline$\gamma$ Virginis & F0 & 1,7 & 1,3 & 2,9 & 6,7 & 4 \\
\hline$\eta$ Arietis & F5 & 1,3 & 1,2 & 2,4 & 6,4 & 2,5 \\
\hline Sol & G2 & 1 & 1 & 2,2 & 5,8 & 1 \\
\hline$\alpha$ Mensae & G5 & 0,93 & 0,93 & 2,1 & 5,6 & 0,8 \\
\hline 70 Ophiuchi A & K0 & 0,78 & 0,85 & 2 & 5,3 & 0,13 \\
\hline 61 Cygni A & K5 & 0,69 & 0,74 & 2 & 4.5 & 0,16 \\
\hline Gliese 185 & M0 & 0,47 & 0,63 & 1,7 & 3,5 & 0,09 \\
\hline
\end{tabular}

Como discutido na Ref.[1], as estrelas devem satisfazer esta condiçãosempre na Seqüência Principal, e assim podemos escrever explicitamente os dois termos da equação 5): o primeiro $E_{\text {grav }} \sim \frac{G M^{2}}{R}$ (o coeficiente para uma esfera é $3 / 5$, mas pode ser ignorado em este tipo de estimativa), e $E_{\text {int }}$ que decorre de multiplicar a energia térmica de uma partícula $k_{B} \bar{T}$ (onde temos escrito $\bar{T}$ para a média espacial da temperatura no interior estelar) pela quantidade de partículas $\frac{M}{m_{p}}$, com $m_{p}$ a massa de um próton (núcleo de hidrogênio). Assim temos que

$$
\frac{G M^{2}}{R} \sim \frac{M}{m_{p}} k_{B} \bar{T}
$$

e poderemos inferir imediatamente a temperatura média de qualquer estrela conhecendo sua massa e raio utilizando esta relação. Por exemplo, para o Sol temos que $\bar{T} \sim 2.2 \times 10^{6} \mathrm{~K}$. Como a energia de um fóton que conse-

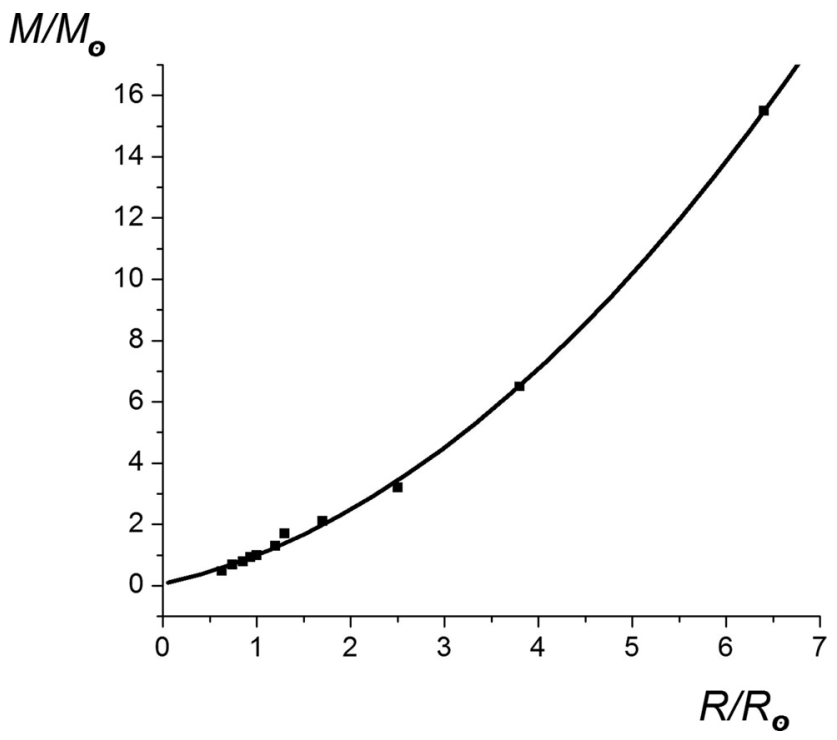

Figura 1: Comparação entre os dados me massas e raios da Tabela 1 (símbolos pontuais) e uma expressão $M \propto R^{2}$ que decorre de supor uma queda na densidade $\propto 1 / r$ (curva cheia). gue ionizar hidrogênio é de $\sim 10^{5} \mathrm{~K}$, vemos que o interior solar deve ser totalmente ionizado, e não composto de gás neutro. Note-se que a relação Virial (6) prescinde totalmente do conhecimento detalhado do interior (distribuição da densidade, transporte de energia etc.), ou seja, sequer é necessário supor que a densidade cai substancialmente na direção da superfície tal como deduzimos anteriormente. E ainda observamos que, devido a que a $\bar{T}$ deve ser menor que a temperatura central $T_{c}$, aquela é um limite inferior para esta última, a qual está relacionada diretamente com as reações nucleares que sustentam a estrutura, tópico de grande interesse que abordaremos a seguir.

Uma segunda característica importante das estrelas é a chamada relação massa-luminosidade. A luminosidade estelar $L$, ou energia emitida por unidade de tempo (ou seja, potência) é diretamente medida conhecendo a distância Dà estrela, e esta última pode ser obtida por vários métodos diferentes. Se não soubermos absolutamente nada das distâncias, o que podemos medir das estrelas é o fluxo de energia $F$ (supondo que ele é carregado na sua totalidade pela luz que detectamos, e não por qualquer outro agente "invisível", tal como neutrinos). Das propostas curriculares do Ensino Médio fica claro que estas duas quantidades estão relacionadas simplesmente por

$$
\frac{L}{4 \pi D^{2}}=F
$$

Tentaremos agora um cálculo simples do fluxo utilizando uma abordagem intuitiva do interior da estrela. Embora sequer tenhamos escrito a equação que determina a temperatura como função da coordenada radial (a qual é análoga às equações (2) e (3)), podemos dizer que o fluxo de energia precisa ser proporcional à diferença de temperaturas. Isto é enfatizado muitas vezes no Ensino Médio, diz-se que o calor passa das regiões quentes para as frias cada vez que há uma diferença $\Delta T$. Mas para se transportar, o calor, representado pelos fótons no interior estelar, estes fótons precisam escoar colidindo com a matéria. Assim, podemos definir um coeficiente que 
contém esta interação e que dificulta ou facilita a passagem dos fótons, tal como uma torneira mais ou menos aberta deixa passar a água. Este coeficiente será chamado de opacidade $\kappa$ (este é o nome que tem na teoria da Estrutura Estelar). Também é apresentado aos alunos do Ensino Médio que a energia dos fótons (radiação) é proporcional à quarta potência da temperatura $\left(a T^{4}\right)$, nas discussões da catástrofe de Rayleigh e Jeans a respeito da distribuição da energia de um corpo negro [10]. Assim, e de forma totalmente fenomenológica, mas que pode ser rigorosamente fundamentada, escrevemos que o fluxo de radiação entre o topo e a base de uma camada esférica deve ter a forma [11]

$$
F=\frac{c t e}{\kappa \rho} \times \frac{\Delta\left(a T^{4}\right)}{\Delta R}
$$

onde termos ainda introduzido a densidade no denominador do primeiro fator, já que se a densidade da matéria atravessada aumentar, o fluxo deve diminuir e vice-versa. Com o mesmo grau de aproximação anterior $\Delta R \sim R$ e utilizando que $\rho \propto M / R^{3}$ e $T \propto M / R$ (da equação Virial (6)), chegamos de imediato a

$$
L \propto F R^{2}=\frac{R^{2}}{\rho} \frac{T^{4}}{R}=\frac{R^{5}}{M} \frac{M^{4}}{R^{5}} \propto M^{3}
$$

desde que $\kappa$ seja considerado constante. Note-se que a equação (9) também não usa nenhuma informação detalhada do interior estelar, mas tão somente o teorema do Virial. Como $R$ se cancela, não é necessária a relação massa-raio nem outro dado de entrada. A Fig. 2 mostra o acordo bem razoável entre uma estimativa básica da luminosidade como função da massa e estas últimas para as mesmas estrelas da Tabela 1, excluídas as duas de

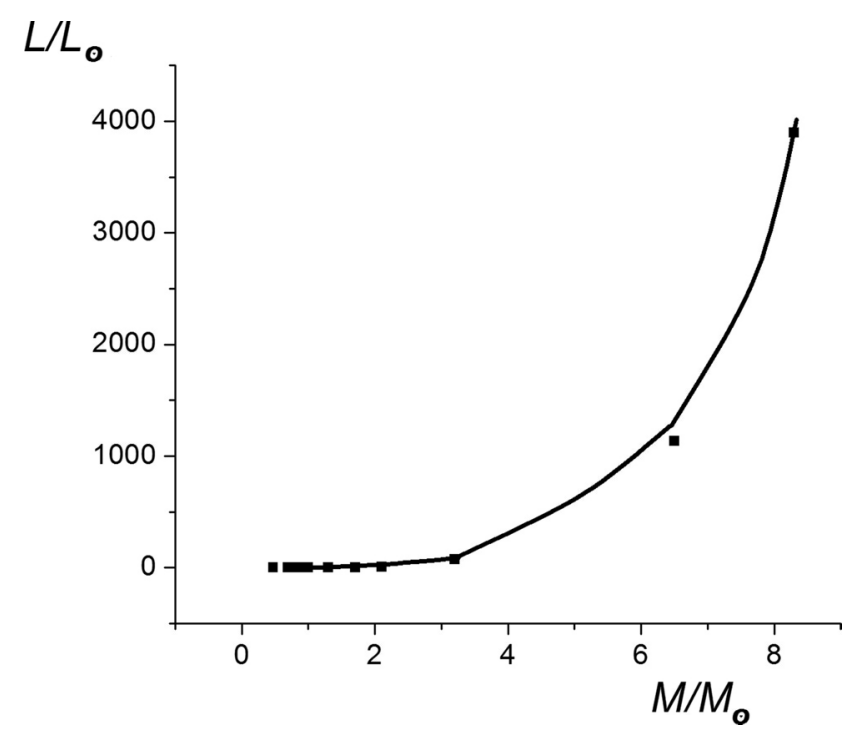

Figura 2: A relação entre a luminosidade e a massa em estrelas de $M \leq 10 M_{\odot}$ da Tabela 1 . A curva sobe rapidamente e resulta um pouco mais empinada que $L \propto M^{3}$, esta última devida à hipótese de opacidade constante e outras simplificações. massas mais altas. Esta exclusão é devida à violação de algumas das hipóteses mais básicas para altas massas, tal como aconteceu na derivação da relação $R \propto M^{1 / 2}$ anteriormente. Mais ainda, como o coeficiente de opacidade $\kappa$ não é constante, mas depende da temperatura, o expoente da relação $L-M$ da Fig. 2 está mais próximo de "4"(este é aproximadamente o expoente real da curva preta) que do "3" da teoria mais simples. Mas é importante enfatizar que este quadro mais simples já é suficiente para ter uma idéia acurada das razões pelas quais as estrelas apresentam as características medidas, no caso $L \propto M^{3}$, com boa aproximação.

Por último, podemos agora apresentar de forma clara o problema da duração da vida das estrelas. Mais de $90 \%$ das estrelas observadas no céu estão na Seqüência Principal, e assim este estágio deve ser muito longo. Em particular, o Sol está há uns 4.5 bilhões de anos nessa fase. Mas a pequena fração de estrelas que já saíram dela permaneceram consumindo o seu hidrogênio um tempo muito variável [11]. De fato, a estimativa mais simples (de novo, sem precisar resolver equações de estrutura etc.) e totalmente geral consiste em dividiro "combustível"disponível (a massa da estrela, suposta toda de hidrogênio) pela taxa de consumo do hidrogênio, responsável pela luminosidade $L$, ou seja

$$
\tau=\frac{M}{L}
$$

Note-se que supomos aqui que todo o hidrogênio pode sofrer fusão, coisa que está longe de ser verdade já que as condições físicas são impossíveis de serem sustentadas para continuar indefinidamentecom a fusão no caroço estelar, e assim grande parte do hidrogênio original da estrela ainda está intocado quando a fusão para (isto é conhecido como o limite de Schoemberg-Chandrasekhar), mas continuaremos sem entrar em estes detalhes.

A equação (10) diz simplesmente que a taxa de consumo de combustível $L$ é o fator mais importante da duração da vida estelar na Seqüência Principal. Mas já vimos que esta luminosidade depende muito da massa $M$, ou seja, as estrelas mais massivas são muito mais luminosas. Isto quer dizer que, embora possuam muito "combustível", o gastam muito mais rapidamente. Utilizando que $L \propto M^{3}$ segundo a equação $\sqrt{9}$, temos de imediato que $\tau \propto 1 / M^{2}$. Pelas mesmas razões apontadas antes, a expressão exata é um pouco mais pronunciada nas massas baixas $\left(\tau \propto 1 / M^{3}\right)$ e um pouco menos nas massas altas $(\tau \propto 1 / M)$, de tal forma que temos obtido uma espécie de média. Esta expressão justifica que as estrelas de maiores massas residem na Seqüência Principal por tempos muito menores que o Sol, da ordem de $\sim$ dezenas de milhões de anos, devido à queima nuclear muito rápida, enquanto as mais leves ainda permanecerão nela por $\sim$ bilhões de anos, já que seu dispêndio de "combustível"é muito vagaroso. Poderíamos apontar um paralelismo com outros sistemas similares: um automóvel de corrida, por exemplo. Como a principal característica 
deve ser a velocidade, o carro precisa ser abastecido logo, já que a potência desenvolvida $(L)$ é muito grande. Por outro lado, um carro "econômico"pretende exatamente o contrário, roda muito com uma quantidade pequena de combustível e assim sua "vida"é muito maior, em correspondência exata com o caso das estrelas.

\section{As reações nucleares no Sol e nas estrelas da Seqüência Principal [11]}

Sendo o hidrogênio o elemento mais abundante da natureza, se formos convidados para "adivinhar" a reação de fusão mais simples no interior estelar, certamente tentaríamos $H+H \rightarrow^{2} H e$. Mas o núcleo de ${ }^{2} H e$ simplesmente não existe, se formado decai imediatamente em dois hidrogênios (a repulsão Coulombiana entre os dois prótons componentes não permite estado ligado). O único estado ligado de dois prótons ou nêutrons que existe é o deuteron ${ }^{2} D$ (o núcleo de deutério, onde um nêutron adicional existe junto ao próton, encontrado na chamada "água pesada"nos reatores nucleares, $D_{2} O$ ) e, para o qual um dos prótons precisa decair em um nêutron por meio de uma reação fraca

$$
H+H \rightarrow{ }^{2} D+\beta^{+}+\nu
$$

onde $\beta^{+}$indica um pósitron e $\nu$ um neutrino que leva embora o momento angular em excesso. Agora bem, este decaimento deve acontecer justamente quando os dois prótons colidem, de tal forma de permitir a formação do deuteron. Levando em conta que uma colisão deste tipo tem uma duração de $\sim 10^{-14} s$ antes das partículas se afastarem demais, é possível ver que esta conversão na equação (11) simultânea com o momento da colisão é muito, mas muito rara. De fato, na maior parte das colisões os dois prótons voltam a se separar sem ter fusionado. A Fig. 3 mostra que somente uma em $10^{28}$ colisões leva efetivamente a fusão e formação do deuteron. Ainda mais, a energia de ligação do deuteron é, porém, pequena, somente $2.2245 \mathrm{MeV}$, ou seja, uma fração de $0.24 \%$ da massa do próton, mas suficiente para fazer que a reação (11) seja exotérmica, já que compensa os 0.42 $\mathrm{MeV}$ de energia cinética média do pósitron e do neutrino, ou os $1.422 \mathrm{MeV}$ se deixarmos o pósitron se aniquilar. Mas o deuteron é realmentemuito frágil: existe uma compensação quase perfeita devida a atração nuclear que compensa a energia cinética inicial e permite esse estado ligado fundamental para a prosseguimento das reações nucleares.

Nos cursos do Ensino Médio é ensinado que a vida média do nêutron livre, determinada pela intensidade da interação fraca, é da ordem de 11 min antes dele decair em um próton. Temos assim, no caso estelar, uma variante que evidencia a "fraqueza"destas interações fracas. É importante destacar que, ao precisar de uma interação fraca, este primeiro estágio produz um longo intervalo temporal para um próton ser convertido em nêutron e passar a formar parte do deuteron. Podemos quantificar esta afirmação escrevendo a vida média do próton nesta conversão, a qual, para as condições do interior solar, resulta $\tau_{p} \approx 7 \times 10^{9}$ anos. Isto quer dizer que são as interações fracas as responsáveis por "esticar" a vida das estrelas para bilhões de anos, se fosse somente pelas forças nucleares e Coulombianas, ou seja,se existisse um "dipróton"ligado, o hidrogênio do Sol fusionaria rapidamente, não em bilhões de anos, mas em frações de segundo (!). Assim, sem as interações fracas as estrelas como o Sol sequer existiriam, explodindo, se formadas, em tempos curtíssimos. Outra forma de dizer o mesmo é que, se nos focarmos em um único próton, este colide bilhões de vezes com outros, e mesmo assim pôde nunca decair e produzir o deuteron que continuará a seqüência de reações. Mas como o número total de prótons total é gigantesco, ordens de grandeza maior que a probabilidade $10^{-28}$ por colisão apontada na Fig. 3, o número de fusões é muito grande e sustenta a estrutura solar embora a cada segundo o Sol irradia ao espaço $3.8 \times 10^{26} \mathrm{~W}$. As estrelas vivem bilhões de anos, e permitem o desenvolvimento de vida nos planetas que as circundam, porque as interações fracas assim o determinam, "regulando"a fusão nuclear para que aconteça de forma vagarosa e não explosiva. Acreditamos que esta conexão da vida das estrelas com o estudo das forças elementares é muito enriquecedora e significativa para discussão em sala de aula.

Uma vez produzido, posteriormente o deuteron $D$ captura um próton, produzindo ${ }^{3} \mathrm{He}$, o qual por sua vez fusiona com outro igual para completar a chamada cadeia chamada de $P P I$

$$
\begin{array}{r}
H+H \rightarrow D+\beta^{+}+\nu \\
D+H \rightarrow{ }^{3} H e+\gamma \\
{ }^{3} H e+{ }^{3} H e \rightarrow{ }^{3} H e+2 H .
\end{array}
$$

Portanto, de forma efetiva houve uma conversão de 4 prótons em um ${ }^{4} \mathrm{He}$, com liberação de energia na forma de neutrinos, pósitrons e gamas. Mas esta "conversão de hidrogênio em hélio"esta longe de ser algo simples (de fato, somente a reação do meio do conjunto (12) é conversão de hidrogênio em hélio !) e vale a pena enfatizar como existem estágios sutilmente "sintonizados"para que isto finalmente aconteça, talvez dando espaço para uma discussão em termos do chamado Princípio Antrópico [12] que mostra um Universo "sintonizado"de tal maneira

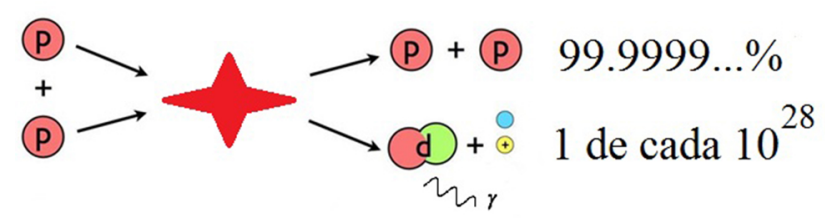

Figura 3: Na maioria esmagadora das colisões os dois prótons emergem intocados porque não podem se ligar. Somente em $1 / 10^{28}$ colisões um deles decai em um nêutron (parte inferior à direita), dando início à cadeia de fusão PPI. 
que permite a existência de observadores humanos que o estudam.

Uma última questão de amplo interesse tem a ver com esta longa duração da vida das estrelas, já justificada mais acima. Sabemos que os céus do Mundo Antigo eram essencialmente os mesmos que vemos hoje. Nada mudou substancialmente depois de vários milênios. Isto quer dizer que o estado das estrelas é notavelmente robusto, ao menos em escalas da civilização humana. Como é que acontece esta estabilidade para as estrelas brilharem (por bilhões de anos, segundo acreditamos)? A resposta é que as estrelas possuem um termostato natural. Um termostato é um dispositivo que se encarrega de manter a temperatura constante. A chave para que o Sol e as estrelas se mantenham estacionárias sem explodir ou colapsar está na relação entre a pressão e a taxa de fusão que libera energia, e que depende bastante da temperatura. Da mesma forma que um termostato aumenta a liberação de energia se a temperatura cai, e a diminui se o sistema esquenta demais, as estrelas reagem de forma análoga.

Suponhamos que a taxa de fusão nuclear aumenta por qualquer razão em uma região do interior da estrela. A seqüência de processos que acontece é

a) a temperatura aumenta por causa de uma maior liberação de energia;

b) como $P V=n R T$, a pressão também aumenta;

c) a maior pressão exercida, o interior se expande (vagarosamente) para manter o equilíbrio hidrostático;

d) assim, a densidade e a temperatura decrescem;

e) a taxa de reações, que depende muito de $T$, decresce e entra de novo em equilíbrio

Agora, se porventura acontecer o contrário, uma diminuição da produção de energia, temos que

a) a temperatura diminui;

b) como $P V=n R T$, a pressão também diminui;

c) com pressão menor, o interior se contrai (vagarosamente) para manter o equilíbrio hidrostático;

d) assim, a densidade e a temperatura crescem;

e) a taxa de reações cresce com a $T$ maior e entra de novo em equilíbrio

Esta descrição é baseada nos fatos físicos básicos e também dispensa qualquer ferramenta matemática sofisticada, e constitui outro argumento importante para uma discussão dos temas da estrutura das estrelas.

\section{Conclusões}

Temos discutido alguns fatos básicos, mas muito relevantes do ponto de vista físico, para levar a discussão à sala de aula do Ensino Médio a respeito da estrutura das estrelas utilizando uma versão algébrica do equilíbrio hidrostático e o equilíbrio Virial. Posteriormente apresentamos um argumento sólido que mostra cómo as interações fracas são determinantes para a vida das estrelas através da regulagem das reações nucleares, mais precisamente, do primeiro estágio no caminho da fusão do hidrogênio, esta última apresentada em geral sem qualquer menção ao decaimento. Estes temas e desdobramentos simples (precedidos, por exemplo, de uma observação do céu estrelado) podem constituir um conjunto de aulas de interesse e relevância para os alunos do Ensino Médio, levando a questionamentos e extensões de importância, e o que é mais importante, a uma apreciação "viva"de alguns tópicos da Física que, embora obrigatórios, aparecem quase sempre descontextualizados. As referências $[1,4,5,7,13]$ contém assim material para o preparo de uma seqüência didática básica que pode ser adaptada ao tempo disponível e necessidades de cada escola, fazendo efetiva a sugestão dos PCN e outras diretrizes.

\section{Referências}

[1] M. Bandecchi, J.E. Horvath e P.S. Bretones, Rev. Bras. Ens. Fís. 41, e20180250 (2019)

[2] BRASIL, Parâmetros Curriculares Nacionais para o Ensino Médio (Ministério da Educação, Brasília, 1998)

[3] SÃO PAULO, Currículo do Estado de São Paulo: Ciências Humanas e suas tecnologias (Secretária da Educação, São Paulo, 2011)

[4] S. Sasson C. da Silva Júnior e P.S.B Sanches, Ciências - Entendendo a Natureza - $6^{\circ}$ Ano, (Ed. Saraiva, São Paulo, 2013), 25 ${ }^{\mathrm{a}}$ ed.

[5] P.S. Bretones Os Segredos do Universo (Ed Atual, São Paulo, 2014), 11 ${ }^{\mathrm{a}}$ ed

[6] Y.P. Erazo, Enseñanza de astronomía estelar a docentes en formación en ciencias naturales. Tese de Doutorado, Universidad del Valle, Cali (2016)

[7] M.B.F. Vieira, Astrofísica estelar no Ensino Médio: análise de uma proposta. Dissertação de Mestrado, Universidade de São Paulo, São Paulo (2018).

[8] A. Sommerfeld, Lectures on Theoretical Physics, Volume 5: Thermodynamics and Statistical Mechanics (Academic Press, Nova Iorque, 1964)

[9] W. Maciel, Introdução à Estrutura e Evolução Estelar (EDUSP, São Paulo, 1999)

[10] https://fisicacontextoaplicacoes.blogspot.com/2016/07/a-fisica-do-seculo-xx.html

[11] J.E. Horvath, Fundamentos de Evolução Estelar, Supernovas e Estrelas Compactas (Ed. Livraria da Física, São Paulo, 2011).

[12] http://www.bertolo.pro.br/fisica_cosmologia/Cosmologia/Cosmology/Anthropic.htm

[13] J.E. Horvath, Rev. Bras. Ens. Fís. 35, 4501 (2013). 